\title{
Failure of Dermal Allograft Repair of Massive Rotator Cuff Tears in Magnetic Resonance Imaging and Clinical Assessment
}

\author{
Conor James Craig Gouk, MBChB, Ryan Mark Shulman, MBBS, Craig Buchan, MBBS (Hons), \\ Michael John Evan Thomas, MBBCh, Fraser James Taylor, MBChB \\ Department of Orthopaedics, Gold Coast University Hospital, Gold Coast, Australia
}

\begin{abstract}
Background: Massive retracted rotator cuff tears represent a therapeutic dilemma, particularly in the young and middle-aged patients who are not appropriate for a reverse total shoulder replacement. Interposition grafting using human dermal allograft offers an alternative treatment.

Methods: A retrospective review of all patients who underwent interposition grafting using human dermal allograft between December 2013 and May 2015 for massive rotator cuff tears at our tertiary referral center was performed. Preoperative and 6 month postoperative magnetic resonance imaging (MRI) assessments were performed in all patients, with postoperative graft integrity being the primary outcome measure. Clinical evaluation was performed using the Oxford shoulder score, Constant score, and Disabilities of the Arm, Shoulder and Hand (DASH) score.

Results: The mean age at the time of follow-up was 54 years. On MRI, 84\% of grafts were seen to have failed at 6 months. Strength was grossly reduced on the operative side when supraspinatus and subscapularis were tested; despite this, Constant score (mean, 48.2) was comparable to that in the previous reports. DASH and Oxford scores were a mean of 24.94 and 37.16, respectively.

Conclusions: Based on these results, in particular the MRI findings, we cannot advocate the use of dermal allograft as an interposition graft for the repair of massive rotator cuff tears.

Keywords: Rotator cuff injuries, Acellular dermal graft tissue, Shoulder, Operative surgical procedure, Magnetic resonance imaging
\end{abstract}

Massive retracted rotator cuff tears represent a therapeutic dilemma, particularly in young and middle-aged patients who are not appropriate for a reverse total shoulder replacement. ${ }^{1-3)}$ Operative options then available to the patient and surgeon are graft repair or reconstruction, superior capsular reconstruction, or tendon transfer. Prior studies have reviewed dermal allograft as an augment to rotator cuff repair; however, augmentation is not possible

Received December 6, 2018; Accepted February 14, 2019

Correspondence to: Conor James Craig Gouk, MBChB

Department of Orthopaedics, Gold Coast University Hospital, 1 Hospital

Blvd, Southport, Gold Coast, Queensland 4215, Australia

Tel: +61-449149889, Fax: +61-7-56874596

E-mail: c.j.gouk.06@aberdeen.ac.uk in many cases as the rotator cuff cannot be mobilized and repaired onto its footprint on the greater tuberosity. ${ }^{4,5}$ Therefore, in such circumstances, interposition grafting using human dermal allograft is a viable therapeutic option. Interposition graft has previously been shown to successfully improve patient's functional ability and decrease pain, without subjecting the patient to the morbidity of tendon transfers or reverse total shoulder arthroplasty. ${ }^{6,7)}$ We aim to determine the outcomes of such cases after dermal allograft interposition graft both clinically and radiologically using magnetic resonance imaging (MRI). 


\section{METHODS}

We present a single-center, two-surgeon, case series of patients treated by the senior authors at Gold Coast Health Campuses between December 2013 and May 2015. Retrospective analysis of those who underwent open rotator cuff repair using human acellular dermal allograft (GraftJacket; Wright Medical, Memphis, TN, USA) for massive rotator cuff tears identified on preoperative MRI assessment was conducted. The Gold Coast Health Human Research Ethics Committee approved this study with consent waived for the retrospective imaging and clinical review (No. HREC/16/QGC/175).

\section{Inclusion and Exclusion Criteria}

Patients with a minimum of 6 months of follow-up who underwent the described treatment for massive rotator cuff tear (defined as greater than $5 \mathrm{~cm}$ in the anteroposterior dimension or involving two or more tendons as per the Snyder Classification ${ }^{8)}$ ) were included. All patients were between 21 and 60 years of age at the time of surgery.

Patients had preoperative MRI demonstrating massive rotator cuff tears, were symptomatic, had failed previous conservative management, and had a functioning deltoid. All were agreeable and able to complete the functional assessments. Any patient with glenohumeral joint arthritis was excluded. Any patient who was found to have a tear amenable to primary repair was excluded. Patients with failed prior primary repair were included; one patient had a previous rotator cuff repair 12 years prior at another institution.

\section{Operative Technique}

The patients were anaesthetized using general anesthetic as well as an infraclavicular block under ultrasound scan guidance. They were placed in the beach chair position using the spider $\mathrm{T}$ max table with a pneumatic arm holder (Smith and Nephew, Andover, MA, USA). An arthroscopic assessment was performed via standard posterior, anterior, and lateral portals. Careful assessment of the chondral surfaces was performed to exclude significant osteoarthritis. The rotator cuff was carefully examined, and mobilization was attempted. If a massive irreparable rotator cuff tear was confirmed, the procedure was then converted to an open procedure via a deltoid splitting approach to allow adequate exposure and facilitate secure fixation to the medial residual tendinous stump. After further mobilization of the supraspinatus, partial repair of the infraspinatus was incorporated into the repair if possible. The GraftJacket was rehydrated and prepared as per manufacturer's instructions, pretensioned before insertion, and subsequently secured as a single layer to the lateral edge of the residual supraspinatus and infraspinatus using multiple interrupted nonabsorbable braided sutures. It was then positioned onto the greater tuberosity. The dermal surface was applied to the footprint and secured using a double-row transosseus equivalent using three medial and two lateral 5.5-mm Healix Advance knotless anchors (Mitek; Depuy Synthes, Raynham, MA, USA). Graft was positioned under slight tension with the arm in zero degrees of abduction and neutral rotation. Then, $3 \mathrm{~mL}$ of $0.3 \mathrm{mg} / \mathrm{mL}$ bioengineered recombinant human platelet-derived growth factor-BB (rhPDGF-BB, Wright Medical) was prepared as per manufacturer instructions and injected around the repair site. Open resection of the AC joint was performed in four patients using an oscillating saw. Biceps tenotomy was performed in three patients and tenodesis in one patient. The long head of biceps was noted to have previously ruptured in two patients and was left intact in one patient. After layered closure, shoulder immobilizer was fitted. A standard postoperative protocol was followed.

\section{Rehabilitation}

During the first three postoperative weeks, patients were allowed to perform pendular and passive shoulder range of motion (ROM) exercises, but between exercises, patients were instructed to keep their arm in a simple shoulder immobilizer similar to a broad arm sling. From 3 weeks, patients were allowed to do isometric exercises and active assisted ROM exercises. From 6 weeks, patients could progress to rotator cuff strengthening exercises in a controlled, gradual manner.

\section{Outcome Measures}

\section{Radiological outcome}

Our primary outcome was MRI appearance of an intact graft. Failure was defined as any retear of the cuff or loss of continuity of the graft at any point. MRI evaluation of the repair was undertaken 6 months postoperatively; preoperative and 6 month postoperative MRI assessments were available for all patients. Assessment was performed via consensus by two fellowship-trained musculoskeletal radiologists with two and five post-fellowship experience each. Goutallier, Tangent, Patte, Thomazeau, and Sagaya classifications were all determined from pre- and postoperative images when appropriate. Anterior to posterior rotator cuff defect at the lateral margin of the acromion, and maximal lateral to medial tendon retraction were recorded. The location of graft failure was documented as 
Clinics in Orthopedic Surgery • Vol. 11, No. 2, 2019•www.ecios.org

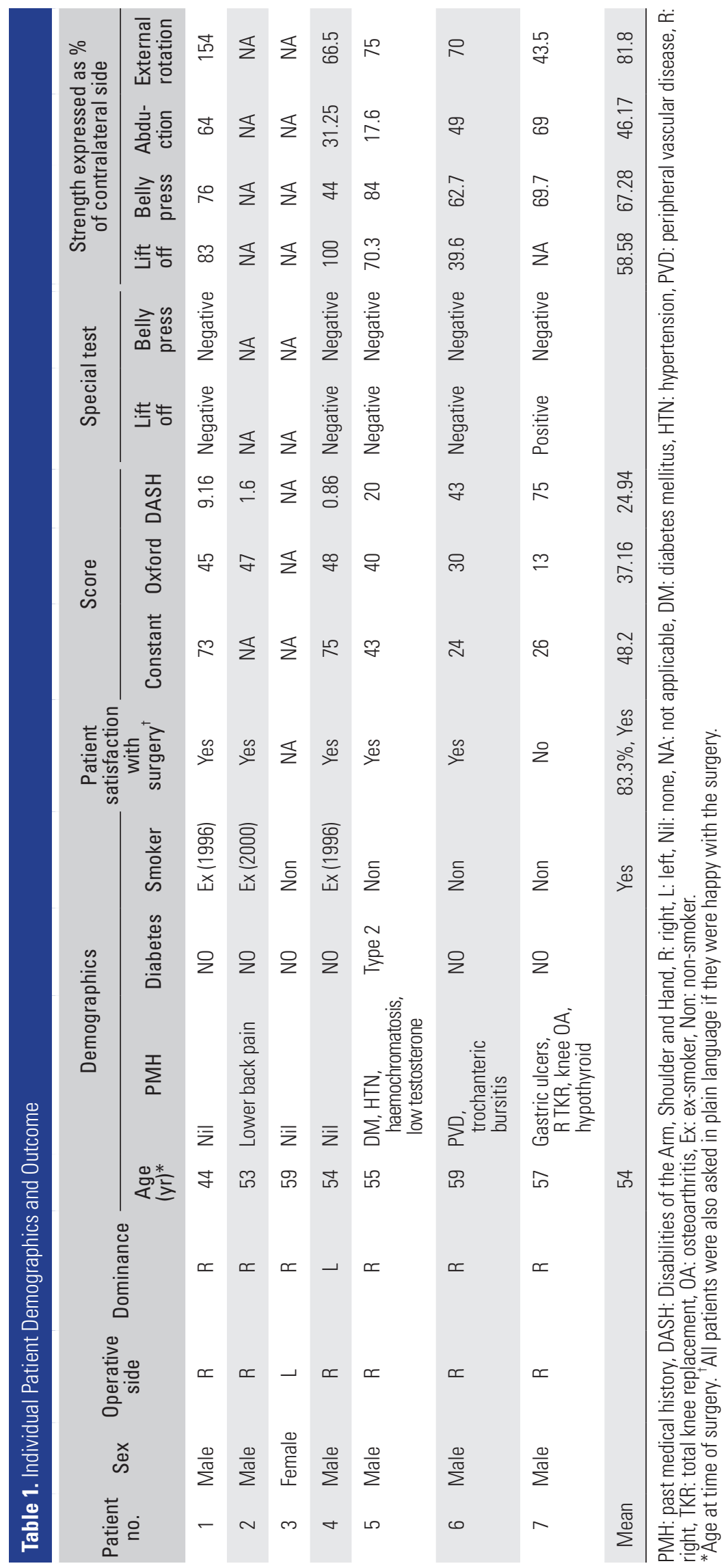


anterior, posterior, medial, lateral, or central.

\section{Clinical outcome}

All eligible patients were evaluated in the clinical setting. Formal clinical evaluation was performed at a mean of 1 year postoperatively, which included strength of abduction, external rotation, belly press, and lift-off tests per our standard shoulder assessment protocol. Oxford, Constant, and Disabilities of the Arm, Shoulder and Hand (DASH) scores were assessed. Strength was measured using an analog hydraulic push-pull dynamometer (Baseline Evaluation Instrument, White Plains, NY, USA) and recorded in

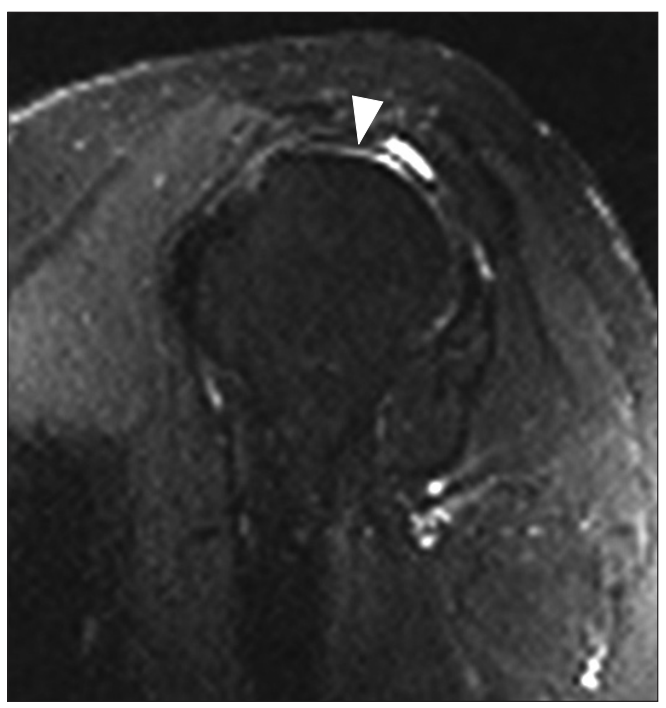

Fig. 1. Proton density fat-saturated sagittal magnetic resonance image demonstrates the typical uniform thin low signal appearance of an intact GraftJacket (arrowhead) traversing the massive rotator cuff tear. pounds; a mean of three attempts was then expressed as a percentage of the contralateral side. All patients were also asked in plain language if they were happy with the surgery.

\section{RESULTS}

Eight consecutive patients (seven males and one female) underwent rotator cuff repair using dermal allograft in an interpositional method. Of these eight, one patient encountered an early surgical infection and subsequently had the interposition graft removed; this patient declined further follow-up.

Of the seven included patients, one patient emigrated after MRI, as such no formal clinical assessment was available; another declined strength measurement and as such complete Constant score could not be obtained. The mean age at the time of surgery was 54 years. Of the seven patients, six were male and all had their right side treated. Only one patient was left-hand dominant. Fifty percent of the patients were no longer working. For one patient, the surgery was a revision of a prior double-row repair, one patient was diabetic, one patient was a smoker at the time of operation and follow-up, and three were ex-smokers preoperatively. This data is summarized in Table 1.

\section{MRI Outcomes}

MRI assessment of the interposition graft showed that only $14 \%$ were completely intact 6 months postoperatively. An intact graft is shown in Fig. 1. MRI demonstrated the location of the graft failure (Table 2). In the majority of cases the failure occurred at the posterior and medial margins (Fig. 2), indicating failure of the graft to integrate into

Table 2. Defect Measurement and Location of Graft Failure as Seen on MRI for Each Patient

\begin{tabular}{|c|c|c|c|c|c|}
\hline \multirow{2}{*}{ Patient no. } & \multicolumn{2}{|c|}{$\begin{array}{l}\text { Anterior to posterior defect (mm), } \\
\text { supraspinatus/infraspinatus }\end{array}$} & \multicolumn{2}{|c|}{ Lateral to medial defect (mm) } & \multirow{2}{*}{ Location of graft failure } \\
\hline & Preoperative & Postoperative & Preoperative & Preoperative & \\
\hline 1 & 36.5 & 26.2 & 37.4 & 34 & Posterior and medial margins \\
\hline 2 & 53.7 & 25.9 & 46.8 & 45.8 & Posterior and medial margins \\
\hline 3 & 25.3 & 16.6 & 23 & 17 & Central, posterior and medial margins \\
\hline 4 & 37.1 & 12.8 & 50.2 & 23.5 & Posterior and medial margins \\
\hline 5 & 32.2 & 19.8 & 45.6 & 24.3 & Posterior margin \\
\hline 6 & 27.7 & 0 & 54.5 & 0 & Intact \\
\hline 7 & 43 & 30.7 & 36.1 & 36.9 & Anterolateral, anterior and posterior margins \\
\hline
\end{tabular}

MRI: magnetic resonance imaging. 


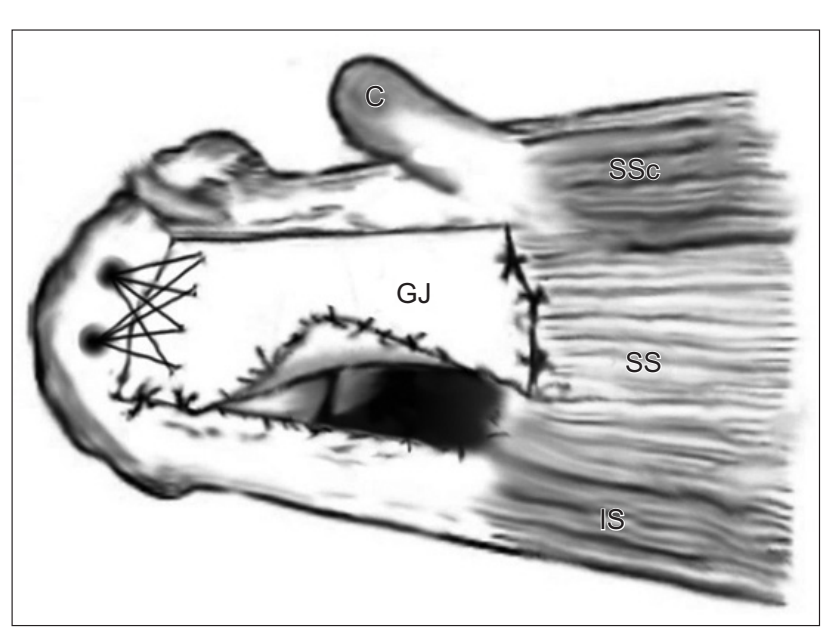

Fig. 2. An axial diagrammatic representation of the most common site of GraftJacket (GJ) failure. The coracoid process (C), subscapularis (SSc), supraspinatus (SS), and infraspinatus (IS) are labelled for orientation.

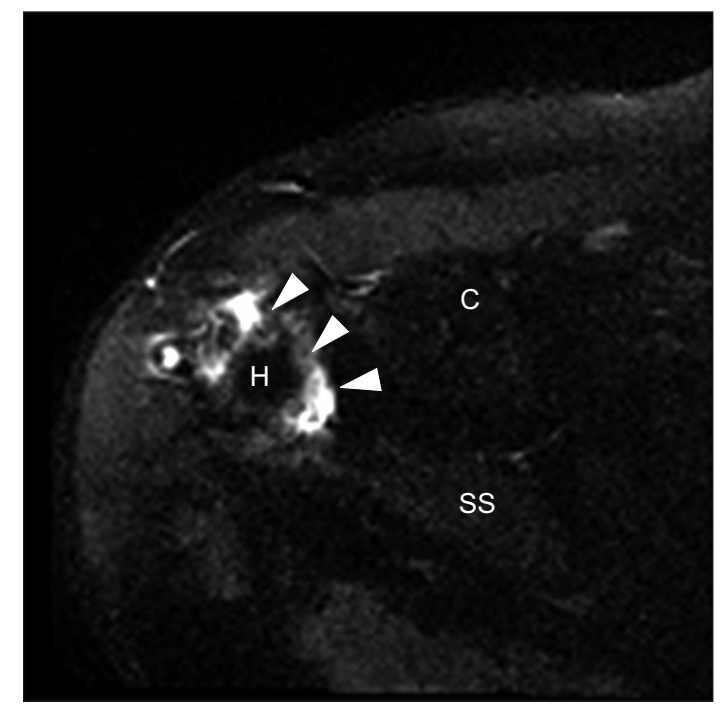

Fig. 3. Proton density fat-saturated axial magnetic resonance image demonstrates the typical anterior displacement of the dehiscent posterolateral GraftJacket margin (arrowheads) uncovering the humeral head $(\mathrm{H})$. The coracoid process (C) and supraspinatus (SS) are labelled for orientation.

the cuff and a failure of the sutures, rather than the insertional anchorage. MRI findings are shown in Figs. 3 and 4.

On MRI, the mean lateral to medial retraction was $41.9 \mathrm{~mm}$ (range, 23 to $54.5 \mathrm{~mm}$ ) preoperatively and 25.9 $\mathrm{mm}$ (range, 0 to $45.8 \mathrm{~mm}$ ) postoperatively. The mean anterior to posterior deficit was measured as $36.5 \mathrm{~mm}$ (range, 25.3 to $53.7 \mathrm{~mm}$ ) preoperatively and $18.85 \mathrm{~mm}$ (range, 0 to $30.7 \mathrm{~mm}$ ) postoperatively. In relation to the musculature and fatty infiltration, at the time of postoperative MRI assessment (6 months), no patient had a change to their

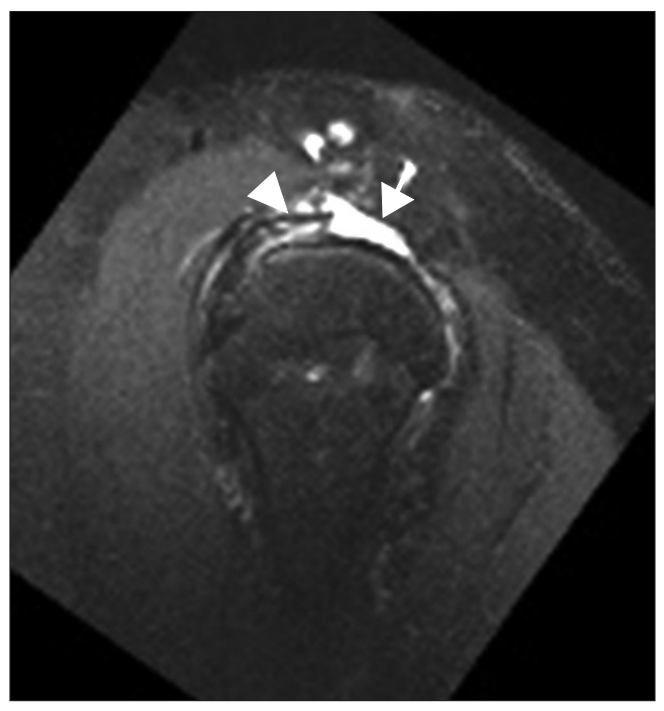

Fig. 4. Proton density fat-saturated sagittal magnetic resonance image demonstrates the GraftJacket (arrowhead) with the posterior deficit (arrow) commonly encountered with failure or dehiscence.

Goutallier score. Preoperatively the mean supraspinatus Goutallier score was 2.57 , and postoperatively it was again 2.57. However, Goutallier, Tangent, Patte, Thomazeau, and Sagaya classifications could not be reliably applied to assess the graft repair due to the nature of the preoperative condition, the repair technique, and their seemingly low sensitivity in this very specific situation.

\section{Clinical Outcomes}

Clinical outcomes are presented in Table 1.

\section{DISCUSSION}

We cannot advocate the use of dermal allograft as an interposition graft for the repair of massive rotator cuff tears based on our results. Functional scores (Oxford, Constant, and DASH scores) appeared to be satisfactory in that these scores were comparable to those in previous published literature reporting successful management of massive rotator cuff tears ${ }^{7)}$ and a high percentage of patients who had undergone the surgery were "happy;" however, our MRI findings suggest the interposition graft fails in $86 \%$ of cases by 6 months. It has been thought that 1 year is required for the shoulder to fully recover; however, it appears the graft fails well before this milestone. $\left.{ }^{6}\right)$ This failure is clinically reflected by the weakness shown, particularly with abduction. Few previous studies reviewing interpositional grafts measured power in a meaningful manner. We have demonstrated that the graft fails at the graft tendon junction, rather than at the site of anchorage. This is similar to 
Gouk et al. Rotator Cuff GraftJacket

Clinics in Orthopedic Surgery • Vol. 11, No. 2, 2019• www.ecios.org

studies looking at other tendinous repairs. ${ }^{9)}$

Moore et al. ${ }^{10)}$ had a failure rate of $100 \%$ seen on MRI at a mean follow-up of 31.3 months. Despite this, they too demonstrated improved functional scores in University of California, Los Angeles (UCLA) shoulder score. Previous studies that can provide a direct comparison to our data showed similar functional scores postoperatively: Modi et al. ${ }^{7)}$ showed a mean Constant score of 42; Rhee and $\mathrm{Oh}^{11)}$ showed a comparable QuickDASH score of 20.9 \pm 3.6 and also a comparable retear or failure rate seen on MRI of $75 \%$ in their "bridging" group at 1 year postoperatively. We think that the functional scores and patientperceived improvement are due to the graft preventing proximal migration and abutment of the humeral head and are likely to be short-lived as the graft becomes thin, macerated, and tears away from the tendinous stump. This degeneration probably occurs due to impingement of the graft against the glenoid in the same way partial thickness articular surface rotator cuff tears occur. ${ }^{12)}$ Further proof exists in that interpositional dermal allograft repairs performed extra-articularly seem to have good outcomes, in pectoralis major repair amongst others. ${ }^{13,14)}$ The intended purpose of the dermal allograft is for the graft to act as a scaffold for normal cellular integration; however, this clearly does not occur in the case of rotator cuff interpositional grafting. ${ }^{5,15,16)}$ Another argument made by Ono et al. ${ }^{17)}$ in their systematic review is that functional outcomes in bridging repairs "closes a hole" but there is "no attempt to restore the length" and as such recovery of power is not achieved.

It could also be argued that the other operative procedures performed, including subacromial bursectomy, biceps tenotomy, and acromioplasty, may be responsible for the patients' improvement. We intend to follow these patients long-term and conduct histological analysis of any extracted repair.

There are several papers that have shown positive radiographic outcomes of interpositional rotator cuff repairs: Bond et al. ${ }^{18)}$ reported a healing rate of $82 \%$ as seen on MRI arthrogram at 1-year follow-up; however, what constituted "healed, intact, partial tear, and failed" was not well described. Generally, in the literature, there is a certain degree of discrepancy on what is defined as failure or retear in the reporting of the graft on postoperative imaging. For example, Badhe et al. ${ }^{19)}$ regarded their porcine acellular graft as intact when it was seen clearly at the insertion site over the greater tuberosity without commenting on the tendinous remnant and graft interface and they documented a failure or retear rate of only $20 \%$. Other studies also have poorly outlined descriptions of their im- aging analysis. Most rely on ultrasound to assess the graft, which can be technically challenging and unfamiliar to most radiologists and/or ultrasonographers. ${ }^{20,21)}$ Gupta et al. ${ }^{22)}$ in 2012 used ultrasound at 3 years postoperatively to assess the interpositional human dermal allograft integrity and noted $74 \%$ remained intact. Then again in 2013, Gupta et al. ${ }^{23)}$ used ultrasound at 2 years postoperatively to assess their porcine dermal interpositional xenograft and noted $73 \%$ remained intact. In both studies, the ultrasound only assessed the graft tendon or graft-humeral interface; the mid-substance was not assessed for tear. According to Duchman et al.s "overview" article ${ }^{24)}$ midsubstance tears relating to the interpositional technique have not been described; however, we observed midsubstance tearing. Also in two separate studies, Gupta et al. ${ }^{22,23)}$ reported that even those who had retear had improvement in pain and function; however, a rudimentary method of strength measure derived from the Modified Medical Research Scale was employed to evaluate power. Notably, in these studies, patients with high degree of fatty infiltration were excluded.

Neumann et al. ${ }^{25}$ ) used the same criteria as Gupta et al. ${ }^{23)}$ with regards to ultrasound assessment of the porcine dermal xenografts graft, showing $91.8 \%$ intact, 3.3\% partial retear, and $4.9 \%$ complete rupture rate (cases were excluded from the analysis if they required arthroplasty within 1 year due to failure). They observed improvement even in those $8.2 \%$ who had failure. Audenaert et al. ${ }^{26)}$ reported on ultrasound assessment of synthetic interposition graft: $90 \%$ of grafts were intact. In both studies of Gupta et al. ${ }^{23)}$ and the studies of Neumann et al. ${ }^{25)}$ and Audenaert et al., ${ }^{26)}$ the patients were in their 60 s.

Jones and Snyder ${ }^{27)}$ assessed human interpositional dermal allograft postoperatively by MR arthrogram. They demonstrated a $15 \%$ retear rate at 3 months and an additional $26 \%$ retear rate at 1 year, and $41.5 \%$ of patients were lost to follow up by 1 year. Ono et al.'s review ${ }^{17)}$ shows the variability of healing rates, $59 \%$ to $90 \%$; despite this, they concluded bridging grafts may be considered. The high healing rates were reported by papers using synthetic grafts made of polyester (Dacron and Mersilene Mesh).

We acknowledge the small sample size as a weakness of this study as well as the large range in clinical measurements. However, we think that the consistent nature of the margins at which the grafts fail is noteworthy and analysis on human subjects has not been performed with this topic. We also note that this study lacks a comparable control group.

Six months postoperative MRI assessment of the graft appears to suggest early failure in the majority of cases, which can be correlated with the low results of 
strength testing. Despite this, the functional outcomes appear comparable with those of previous studies that advocate the use of dermal allograft. We believe the acceptable functional outcome will be short-lived given the MRI outcomes. We will continue to follow up on these patients and review their long-term functional outcomes and pattern.

This research highlights the difficulty of treating massive rotator cuff tears. Our radiological findings provide further support that this treatment option has high failure rates for this difficult clinical condition. We have demonstrated a very high rate of failure and the low healing rates with this option. Superior capsular reconstruction may provide an improvement on this technique and we will await further reports on this technique.

\section{CONFLICT OF INTEREST}

No potential conflict of interest relevant to this article was reported.

\section{REFERENCES}

1. Gerber C, Fuchs B, Hodler J. The results of repair of massive tears of the rotator cuff. J Bone Joint Surg Am. 2000;82(4):505-15.

2. Sugaya $H$, Maeda K, Matsuki K, Moriishi J. Repair integrity and functional outcome after arthroscopic double-row rotator cuff repair: a prospective outcome study. J Bone Joint Surg Am. 2007;89(5):953-60.

3. Zumstein MA, Jost B, Hempel J, Hodler J, Gerber C. The clinical and structural long-term results of open repair of massive tears of the rotator cuff. J Bone Joint Surg Am. 2008;90(11):2423-31.

4. Agrawal V. Healing rates for challenging rotator cuff tears utilizing an acellular human dermal reinforcement graft. Int J Shoulder Surg. 2012;6(2):36-44.

5. Ely EE, Figueroa NM, Gilot GJ. Biomechanical analysis of rotator cuff repairs with extracellular matrix graft augmentation. Orthopedics. 2014;37(9):608-14.

6. Kokkalis ZT, Mavrogenis AF, Scarlat M, et al. Human dermal allograft for massive rotator cuff tears. Orthopedics. 2014;37(12):e1108-16.

7. Modi A, Singh HP, Pandey R, Armstrong A. Management of irreparable rotator cuff tears with the GraftJacket allograft as an interpositional graft. Shoulder Elbow. 2013;5(3):188-94.

8. Millstein ES, Snyder SJ. Arthroscopic evaluation and management of rotator cuff tears. Orthop Clin North Am. 2003;34(4):507-20.

9. Sclamberg SG, Tibone JE, Itamura JM, Kasraeian S. Sixmonth magnetic resonance imaging follow-up of large and massive rotator cuff repairs reinforced with porcine small intestinal submucosa. J Shoulder Elbow Surg. 2004;13(5):538-41.

10. Moore DR, Cain EL, Schwartz ML, Clancy WG Jr. Allograft reconstruction for massive, irreparable rotator cuff tears. Am J Sports Med. 2006;34(3):392-6.
11. Rhee SM, Oh JH. Bridging graft in irreparable massive rotator cuff tears: autogenic biceps graft versus allogenic dermal patch graft. Clin Orthop Surg. 2017;9(4):497-505.

12. Modi CS, Smith CD, Drew SJ. Partial-thickness articular surface rotator cuff tears in patients over the age of 35: etiology and intra-articular associations. Int J Shoulder Surg. 2012;6(1):15-8.

13. Dehler T, Pennings AL, ElMaraghy AW. Dermal allograft reconstruction of a chronic pectoralis major tear. J Shoulder Elbow Surg. 2013;22(10):e18-22.

14. Rao BM, Kamal TT, Vafaye J, Taylor L. Surgical repair of hip abductors: a new technique using Graft Jacket allograft acellular human dermal matrix. Int Orthop. 2012;36(10):204953.

15. Acevedo DC, Shore B, Mirzayan R. Orthopedic applications of acellular human dermal allograft for shoulder and elbow surgery. Orthop Clin North Am. 2015;46(3):377-88.

16. Shea KP, Obopilwe E, Sperling JW, Iannotti JP. A biomechanical analysis of gap formation and failure mechanics of a xenograft-reinforced rotator cuff repair in a cadaveric model. J Shoulder Elbow Surg. 2012;21(8):1072-9.

17. Ono Y, Davalos Herrera DA, Woodmass JM, Boorman RS, Thornton GM, Lo IK. Graft augmentation versus bridging for large to massive rotator cuff tears: a systematic review. Arthroscopy. 2017;33(3):673-80.

18. Bond JL, Dopirak RM, Higgins J, Burns J, Snyder SJ. Arthroscopic replacement of massive, irreparable rotator cuff tears using a GraftJacket allograft: technique and preliminary results. Arthroscopy. 2008;24(4):403-9.

19. Badhe SP, Lawrence TM, Smith FD, Lunn PG. An assessment of porcine dermal xenograft as an augmentation graft in the treatment of extensive rotator cuff tears. J Shoulder Elbow Surg. 2008;17(1 Suppl):35S-39S.

20. Prickett WD, Teefey SA, Galatz LM, Calfee RP, Middleton WD, Yamaguchi K. Accuracy of ultrasound imaging of the 
Gouk et al. Rotator Cuff GraftJacket

Clinics in Orthopedic Surgery • Vol. 11, No. 2, $2019 \bullet$ www.ecios.org

rotator cuff in shoulders that are painful postoperatively. J Bone Joint Surg Am. 2003;85(6):1084-9.

21. Mack LA, Nyberg DA, Matsen FR 3rd, Kilcoyne RF, Harvey D. Sonography of the postoperative shoulder. AJR Am J Roentgenol. 1988;150(5):1089-93.

22. Gupta AK, Hug K, Berkoff DJ, et al. Dermal tissue allograft for the repair of massive irreparable rotator cuff tears. Am J Sports Med. 2012;40(1):141-7.

23. Gupta AK, Hug K, Boggess B, Gavigan M, Toth AP. Massive or 2-tendon rotator cuff tears in active patients with minimal glenohumeral arthritis: clinical and radiographic outcomes of reconstruction using dermal tissue matrix xenograft. Am J Sports Med. 2013;41(4):872-9.

24. Duchman KR, Mickelson DT, Little BA, et al. Graft use in the treatment of large and massive rotator cuff tears: an overview of techniques and modes of failure with MRI correlation. Skeletal Radiol. 2019;48(1):47-55.

25. Neumann JA, Zgonis MH, Rickert KD, et al. Interposition dermal matrix xenografts: a successful alternative to traditional treatment of massive rotator cuff tears. Am J Sports Med. 2017;45(6):1261-8.

26. Audenaert E, Van Nuffel J, Schepens A, Verhelst M, Verdonk R. Reconstruction of massive rotator cuff lesions with a synthetic interposition graft: a prospective study of 41 patients. Knee Surg Sports Traumatol Arthrosc. 2006;14(4):360-4.

27. Jones CR, Snyder SJ. Massive irreparable rotator cuff tears: a solution that bridges the gap. Sports Med Arthrosc Rev. 2015;23(3):130-8. 